\title{
Religious Coping as Moderator of Psychological Responses to Stressful Events: A Longitudinal Study
}

\author{
Felipe E. García ${ }^{1, *}$, Darío Páez ${ }^{2}$, Alejandro Reyes-Reyes ${ }^{1}$ and Rodolfo Álvarez ${ }^{1}$ \\ 1 Facultad de Ciencias Sociales, Universidad Santo Tomás, Concepción 4061501, Chile; \\ areyesr@santotomas.cl (A.R-R.); rodolfoalvarez@santotomas.cl (R.Á.) \\ 2 Department of Social Psychology and Methodology, University of the Basque Country, San Sebastian 20018, \\ Spain; dario.paez@ehu.es \\ * Correspondence: fgarcia@santotomas.cl
}

Academic Editor: Paweł Marian Socha

Received: 1 February 2017; Accepted: 2 April 2017; Published: 7 April 2017

\begin{abstract}
The aim of this study was to evaluate the association of positive and negative religious coping with posttraumatic symptoms (PTS) and growth (PTG). Their moderating role was also examined among predictors such as social support and the subjective severity of event with PTS and PTG. Two hundred and eleven Chilean adults (58.3\% women) of 18 years and older who had been exposed to highly stressful were surveyed. The Brief-RCOPE, the Brief-COPE subscale of social support, the Subjective Severity of Event Scale, and a socio-demographic questionnaire were used as measurements at time one. The Posttraumatic Growth Inventory-short form (PTGI-SF) and Short Posttraumatic Stress Disorder Rating Interview (SPRINT-E) was used to collect baseline scores and six months after. The results show that negative religious coping predicts the increase in PTS, positive religious coping predicts the increase in PTG and plays a moderation role: at low levels of positive religious coping it was found a strong association between coping by seeking social support and PTG, while at high level the association is weak. These results are discussed in the framework of the functionality of positive and negative religious coping and its role in adjusting to potentially traumatic events.
\end{abstract}

Keywords: posttraumatic growth; posttraumatic symptoms; social support; subjective severity; posttraumatic stress disorder

\section{Introduction}

This article examines the role of religiosity and religious coping $(\mathrm{RC})$ when adjusting to traumatic events, both in attenuation of symptoms and favouring positive effects of growth. We will discuss the relationship between positive and negative $\mathrm{RC}$ with social support and its role both in symptoms of trauma and posttraumatic growth. Reviewed studies suggest the potential role of RC as a moderator between adjustment and others forms of coping like social support and also suggest that RC is different from other forms of coping-like coping by seeking social support.

There is evidence indicating that religiosity helps to cope with highly stressful or potentially traumatic events. For example, religious beliefs can help in re-evaluating stressful situations such as a mild threat or a challenge [1], or help in attributing meaning to them [2]. Stressful or traumatic experiences can, in turn, lead to a deepening of religiosity [1].

One of the probable positive consequences after living through an extreme event is posttraumatic growth (PTG), defined as the perception of positive changes in self and in relations with others, as a result of the struggle needed to deal with the consequences of a highly stressful or traumatic event [3]. Meta-analyses by Helgeson, Reynolds and Tomich [4] and by Prati and Pietrantoni [5] identify religiosity as one of the factors associated to the development of PTG. For example, a positive 
relationship has been found between RC and PTG in patients with cancer [6] and in survivors of a natural disaster [7-9]. However, Pargament, Feuille and Burdzy [10] warn that RC can also lead to harmful results. For this reason, the investigation must recognize 'the bitter' as much 'the sweet' in religious attitudes. In the same vein, Pargament, Smith, Koenig and Perez [11] differentiate between positive and negative RC. The former leads to psychological and spiritual satisfaction, based on spiritual support, religious purification, looking for help from priests or church members and religious forgiveness. The latter refers to a punitive religious appreciation, spiritual unrest and interpersonal religious unrest. Studies show a different relationship between positive and negative RC with the psychological effects that occur after a stressful traumatic event. In one of the first studies regarding this field, Koenig, Pargament and Nielsen [12] observed that those who had a negative religious view showed low physical health and higher levels of depression. Conversely, a positive religious view was linked to a more positive mental health. Other longitudinal investigations have shown that an increased spiritual questioning at the onset of a negative experience is a predictor of a significant increase in the indexes of psychological disorders over time [13,14]. The study by Pargament et al. [14] also shows us that positive RC is associated to improvements in health, and negative RC is associated with the decrease of health over time. In the study by Harris et al. [15], negative RC showed a significant positive relationship with posttraumatic symptoms (PTS) both immediately after and a year later. On the other hand, positive RC did not show a relationship with PTS in any of the two instances. While PTS have a direct relationship with negative RC [16-19], positive RC is associated to an increase of PTG as time passes [14]. Other studies have shown that PTG has a direct relationship with positive RC $[1,8,20]$. For example, in the study performed by Ai, Hall, Pargament and Tice [21] with heart patients, it was observed that positive pre-operation RC predicts PTG thirty months after operation.

Prominent among the non-religious psychosocial factors and coping strategies associated to psychological responses after a stressful event are the perceived level and seeking of social support or asking and receiving emotional, instrumental and informative help from others to deal with stress. Social support is defined as the perceived or actual group of instrumental and expressive dispositions, which are emitted by various sources [22]. Social support decreases the cognitive impact of stressful events, it allows a better regulation of negative emotions, it favours the control of dysfunctional conducts, reinforces distraction and gratification activities, and it helps to better solve practical problems, which jointly contributes to an increase in PTG—-both social support and coping by seeking social support were associated to PTG in a meta-analysis [5]. It has also been postulated that an increase in social support could be one of the processes by which religious coping and religiosity are positively associated with adjusting to stress and experiencing less symptomatology [23]. Two studies have confirmed this association by showing that the more negative RC, the less social support [24], and that the higher RC, the higher social support [8]. The studies of Avilés, Cova, Bustos and García [25] and the authors [8] have suggested that social support is not the only mechanism by which RC has a positive effect such as PTG. These studies showed that positive RC is associated to PTG even after controlling for social support. This evidence showed that religiosity helps growth beyond its function as a provider of social networks that are supportive in the spiritual area, as other studies have suggested [21]. Both studies also showed that positive RC was a stronger predictor of PTG than social support.

Perceiving an event as severe also has an important influence in the psychological responses to stressful events. An association has been observed between severity and PTS [26,27]. A similar association has also been observed between subjectively perceived severity and PTG in a meta-analysis [5] and in two studies with survivors of an earthquake $[8,28]$. This is a population in which Shakespeare-Finch and Lurie-Beck [29] have found positive relationships between PTS and PTG without finding the same association when exposed to other types of events. The influence of the severity of event on psychological responses also appears to be mediated [8] or moderated [30] by RC.

Thus, it seems especially interesting to evaluate the role of religiosity as a mediator or moderator between predicting variables and psychological responses. It has been observed that negative RC acts as a process that explains the increase of symptoms and hinders growth. For example, it mediated 
between the experience of childhood abuse and PTS [31], it dampened the relationship between direct traumatic events and PTG [32], and it strengthened the relationship between stress and depression [33]. Positive RC also acts as a mediator or an explanatory process, but in an opposite way, because it partially mediated the relationship between gender and PTG [16], or it totally mediated the relationship between subject severity and PTG [8].

With this background, we hypothesize that severity of event will be associated with PTS and PTG, positive RC and perceived social support will be associated to PTG, while RC and low social support will be associated to PTS. We also expect that positive RC will moderate the relationship between social support and the change in PTG level and that the negative RC will moderate the relationship between subjective severity and the change in PTS levels.

\section{Method}

\subsection{Design}

This is a quasi-longitudinal survey design, because measures were taken on two occasions, with six months' difference between them.

\subsection{Participants}

Two hundred and eleven adults of general population participated in this study, inhabitants of Concepcion province in Chile, who have experienced a highly stressful event in the last four years. Women represented $58.3 \%$ and men $41.7 \%$, aging from 18 to 83 years old $(M=34.93 ; D E=13.92)$. Regarding education, $65.2 \%$ had university studies (complete or incomplete), 30.1\% had high school studies (complete or incomplete), and $4.7 \%$ had primary school (complete or incomplete).

\subsection{Instruments}

List of potentially traumatic event. A dichotomic (yes/no) question was included, asking if the person has experienced an extremely negative event in the last four years-based on Norris, Hamblen, Brown and Schinka [34]. This list included ten events: natural disaster, serious personal illness, serious illness of a loved one, accident at home, accident at the workplace, death of a loved one, breakup, police violence, domestic violence and criminal violence, and an open question ('other, specify'). All these events involve a potential threat to one's life or someone close to the participant.

Posttraumatic Growth Inventory, short form (PTGI-SF), was developed by Cann et al. [35] to measure PTG. It consists of 10 items that are answered in a Likert scale from 0 ('I did not experience this change') to 5 ('I experienced this change to a very great degree'). It was validated for the Chilean population by the authors [36], showing an appropriate internal consistency $(\alpha=0.94)$, a high correlation with the total PTGI scale $(r=0.98)$ and construct validity, replicating the factorial structure of the original version.

Short Posttraumatic Stress Disorder Rating Interview (SPRINT-E), was elaborated by Norris et al. [34] to measure posttraumatic symptoms. It consists of 12 items that are answered in a Likert scale from nothing $=0$ to extremely $=4$. It was validated for Chile by Leiva-Bianchi and Gallardo [37]. The scale obtained an adequate internal consistency $(\alpha=0.92)$, concurrent validity when correlating with the Davidson trauma scale and construct validity.

Brief-RCOPE was developed by Pargament et al. [11] to evaluate RC, and was translated to Spanish by Rivera and Montero [38]. It has two sub-scales of 7 items each: Positive RC and Negative RC. It is answered in a Likert scale from 1 ('not at all') to 3 ('a great deal'). The instrument has reliability and validity in different populations and with different stressors [10]. Additionally, Rivera and Montero obtained adequate reliability for Latin American populations for both dimensions, apart from convergence validity with other scales of religiosity. In a study performed by the authors [8] with Chilean population, an $\alpha=0.95$ was obtained for positive RC, 0.90 for negative RC, and 0.90 for negative RC. 
Brief-COPE was developed by Carver [39] to measure coping strategies, and was translated to Spanish and validated for Spanish by Morán, Landero and González [40]. Subscales of instrumental social support and emotional support were used for the evaluation of social support with a global score because previous factor analysis found a one dimension solution [40]. This scale consists of 4 items that are answered in a Likert scale from 0 ('not at all') to 3 points ('much'). In the study by Moran et al. [40], this scale obtained an internal consistency of $\alpha=0.74$.

Subjective Severity of Event Scale consists of three questions to evaluate if the person perceives an event as traumatic, serious or extremely upsetting [41]. Each question is answered in a Likert scale from 0 ('not at all') to 4 ('extremely'). The scale obtained an $\alpha=0.84$ in the study by Alzugaray et al. [41].

Socio-demographic questionnaire included questions regarding gender, age and educational level.

\subsection{Procedure}

A successive sampling with a panel sampling plan was established. An initial random sample $(\mathrm{N}=470)$ of the general population was enrolled by phone call from the census, and 211 people that declared in the first call having experienced a highly stressful event in the last four years were included in the final sample [42]. This sample was interviewed twice. The participants were interviewed between February and April 2015 by two trained psychology students. Questionnaires were self-administered but in the presence of the interviewers. There was no refusal to the participation. Six months after, the participants were interviewed again by the same collaborators. In that occasion, five people ( $2 \%$ of the original sample) were not found: one had died, three had moved to another city and one did not answer his or her phone. These people were excluded from the analysis.

Before applying the surveys, the participants were asked to sign an informed consent form in which their willingness and the confidentiality of the data were stated. The study was evaluated and approved by the Ethics Committee of Universidad Santo Tomás ( $\left.{ }^{\circ} 89 / 2014\right)$.

\subsection{Data Analysis}

First, a descriptive analysis of the variables of the study was performed. Then, the values of the main dependent variables were calculated, i.e. changes in the PTG and PTG score from time 1 (T1) to time 2 (T2). For this, we used the strategy followed by Gottman and Krokoff [43], which consists in subtracting the scores obtained at T1 from the scored obtained at T2 for each variable. In this way, a range of scores was obtained, from negative values when PTG and PTS declined, to positive values that reflected an increase in the levels of PTG and PTS.

The Pearson product moment correlation coefficient (Pearson r) was used to evaluate the relationship between the study variables. Then, Hierarchical Linear Regressions were performed to evaluate the influence of interdependent social support variables, subjective severity and RC at T1 on changes in PTG and PTS, incorporating the hypothesized interactions in the last step. Finally, we performed a bias-corrected bootstrap estimation (10,000 samples) with a confidence interval of $95 \%$ to evaluate the moderating role of positive RC between social support and the change in PTG, and the moderating role of negative RC between subjective severity and change in PTS. For the analysis, SPSS 20 statistic software and PROCESS for SPSS [44] programs were employed.

\section{Results}

Regarding the stressful events, $22.7 \%$ mentioned the death of a loved one, $13.7 \%$ mentioned a natural disaster, $11.4 \%$ was affected by the serious disease of a loved one, $10.4 \%$ was affected by a breakup and $41.8 \%$ was affected by other stressful events such as a serious illness, an accident at home, a workplace accident, a car accident, violence from the state, violence at home or criminal violence, among others.

The results of the descriptive statistics of the variables (see Table 1) showed asymmetry coefficients and curtosis lower than \pm 2.0 , which indicates similarity with the normal curve [45]. The internal 
consistency of the scales, established by Cronbach alpha coefficient, ranged between 0.82 (social support) and 0.95 (positive RC).

Table 1. Descriptive statistics of the study variables $(n=211)$.

\begin{tabular}{cccccccc}
\hline & Minimum & Maximum & Mean & SD & Skewness & Kurtosis & $\alpha$ \\
\hline Subjective & 0 & 12 & 6.59 & 3.09 & -0.24 & -0.69 & 0.86 \\
severity T1 & 0 & 12 & 5.58 & 3.33 & 0.14 & -0.85 & 0.82 \\
Social support T1 & 0 & 21 & 8.82 & 6.94 & 0.19 & -1.25 & 0.95 \\
Positive RC T1 & 0 & 15 & 2.69 & 3.61 & 1.46 & 1.58 & 0.83 \\
Negative RC T1 & 0 & 36 & 10.37 & 7.44 & 0.80 & 0.77 & 0.91 \\
PTS T1 & 0 & 50 & 26.57 & 12.77 & -0.43 & -0.62 & 0.92 \\
PTG T1 & 0 & 35 & 9.48 & 7.41 & 0.65 & 0.30 & 0.92 \\
PTS T2 & 0 & 50 & 25.15 & 12.17 & -0.05 & -0.55 & 0.94 \\
PTG T2 & 0 & &
\end{tabular}

RC: Religious coping; PTS: Posttraumatic symptoms; PTG: Posttraumatic growth.

A Student t-test was performed for independent samples to observe if there were differences due to gender in the change of PTG and PTS. We did not observe differences, which is why gender was excluded as a control variable in hierarchical regressions.

Then, we explored the associations between subjective severity of event, positive and negative RC, social support, PTG, PTS. We also looked at how severity of event, RC and social support in Time 1 predict changes in PTG and PTS or differences between Time 1 and Time 2 PTG and PTS. The results are observed on Table 2.

Table 2. Pearson r correlations between the study variables $(n=211)$.

\begin{tabular}{lccccccccc}
\hline & $\mathbf{2}$ & $\mathbf{3}$ & $\mathbf{4}$ & $\mathbf{5}$ & $\mathbf{6}$ & $\mathbf{7}$ & $\mathbf{8}$ & $\mathbf{9}$ & $\mathbf{1 0}$ \\
\hline 1. Subjective severity T1 & $0.16^{*}$ & $0.17^{*}$ & $0.24^{* * *}$ & 0.13 & 0.07 & 0.07 & $0.44^{* * *}$ & $0.26^{* * *}$ & $0.18^{* *}$ \\
2. Positive RC T1 & - & 0.09 & $0.27^{* * *}$ & $0.49^{* * *}$ & $0.30^{* * *}$ & $0.22^{* * *}$ & 0.10 & 0.07 & 0.04 \\
3. Negative RC T1 & & - & 0.11 & -0.03 & 0.08 & -0.11 & $0.40^{* * *}$ & $0.31^{* * *}$ & 0.09 \\
4. Social support T1 & & & - & $0.27^{* * *}$ & $0.14^{*}$ & $0.15^{* *}$ & $0.27^{* * *}$ & 0.13 & $0.15^{*}$ \\
5. PTG T1 & & & & - & $0.53^{* * *}$ & $0.52^{* * *}$ & 0.09 & 0.01 & 0.08 \\
6. PTG T2 & & & & & - & $-0.45^{* * *}$ & 0.07 & 0.04 & 0.03 \\
7. Change in PTG & & & & & & - & 0.02 & -0.02 & 0.05 \\
8. PTS T1 & & & & & & - & $0.49^{* * *}$ & $0.51^{* * *}$ \\
9. PTS T2 & & & & & & $-0.50^{* * *}$ \\
10. Change in PTS & & & & & & - \\
\hline
\end{tabular}

The table shows that subjective severity was unrelated with PTG in any case (T1, T2 and with changes scores), but severity had a significant relationship with PTS in general-at T1, T2 and with changes scores. In turn, positive RC shows a significant relationship with PTG at T1 and T2 and also positive RC at Time 1 was associated to the increase of PTG at T2. Positive RC does not show a significant relationship with PTS in any case. On the other hand, negative RC shows a significant relationship with PTS at T1 and T2, and predicts the increase in PTS. Negative RC does not have a significant relationship with PTG in any of the measurements. Coping by seeking social support shows positive and significant relationships with PTG in all cases. Globally, positive RC and social support were associated to PTG and unrelated to PTS. Conversely, negative RC was associated to PTS and unrelated to PTG.

Then, regressions were performed to predict the change (increase or decrease) of PTG and PTS. First, it was evaluated if RC influenced change in PTG, controlling for social support coping. For this reason, social support coping was included in the first step and positive and negative RC were incorporated in the second step. To evaluate a possible moderation, the interaction between social support and RC was included in a third step. The results show a significant model for the three steps, the third with a value $\mathrm{F}(4206)=6.378, p<0.001$; with an $\mathrm{R}^{2}=0.11$ and a significant increase in explained 
variance of five percent-but in any case, effect of predictors or explained variance is not so strong. The results are shown on Table 3. When RC was included in the second step, the positive association of coping by social support with the increase of PTG was no longer significant, while the negative RC decreases PTG, the positive RC increases PTG. In the third step, the three variables predict the change in PTG.

Table 3. Hierarchical regression on the change in posttraumatic growth $(n=211)$.

\begin{tabular}{|c|c|c|c|c|c|c|}
\hline & & \multicolumn{2}{|c|}{$\begin{array}{l}\text { Unstandardized } \\
\text { Coefficients }\end{array}$} & \multirow{2}{*}{$\begin{array}{c}\begin{array}{c}\text { Typified } \\
\text { Coefficients }\end{array} \\
\text { B }\end{array}$} & \multirow[t]{2}{*}{$\mathrm{T}$} & \multirow[t]{2}{*}{$p$} \\
\hline & & B & SE & & & \\
\hline \multirow[t]{3}{*}{ Step 1} & $\mathrm{R}^{2}=0.02 . \Delta \mathrm{R}^{2}=0.02, p=0.034$ & & & & & \\
\hline & (Constant) & -1.55 & 1.62 & & -0.959 & 0.339 \\
\hline & Social support $\mathrm{T} 1$ & 0.53 & 0.25 & 0.15 & 2.140 & 0.034 \\
\hline \multirow[t]{5}{*}{ Step 2} & $\mathrm{R}^{2}=0.08 . \Delta \mathrm{R}^{2}=0.06, p=0.003$ & & & & & \\
\hline & (Constant) & -2.60 & 1.77 & & -1.467 & 0.144 \\
\hline & Social support $\mathrm{T} 1$ & 0.39 & 0.25 & 0.11 & 1.530 & 0.127 \\
\hline & Positive RC T1 & 0.35 & 0.12 & 0.20 & 2.890 & 0.004 \\
\hline & Negative RC T1 & -0.46 & 0.23 & -0.14 & -2.047 & 0.042 \\
\hline \multirow[t]{6}{*}{ Step 3} & $\mathrm{R}^{2}=0.11 . \Delta \mathrm{R}^{2}=0.05, p=0.005$ & & & & & \\
\hline & (Constant) & -7.03 & 2.33 & & -3.021 & 0.003 \\
\hline & Social support $\mathrm{T} 1$ & 1.29 & 0.40 & 0.35 & 3.216 & 0.002 \\
\hline & Positive RC T1 & 0.91 & 0.23 & 0.52 & 3.982 & 0.000 \\
\hline & Negative RC T1 & -0.55 & 0.22 & -0.16 & -2.437 & 0.016 \\
\hline & Social support $\times$ Positive $R C$ & -0.10 & 0.03 & -0.49 & -2.871 & 0.005 \\
\hline
\end{tabular}

RC: Religious coping.

The significant interaction of positive RC and social support implies a moderation effect of positive RC on the relation between social support and change in PTG. Regarding these moderation effects in Table 4, a low level of positive RC is associated to a significant and positive association of social support with an incremental change in PTG, while at a high level of positive RC level social support was not associated to change in PTG (see Figure 1). When positive RC is low, social support was strongly associated to the increase of PTG; when positive RC is moderate, the relationship between support and change is positive but weaker; when positive RC is high, there is no relationship between social support level and PTG changes. In other words, social support has a strong influence on the change in PTG when positive RC is low.

Table 4. Conditional effects of social support coping in the change posttraumatic growth for specific values of positive religious coping $(n=211)$.

\begin{tabular}{ccccccc}
\hline \multirow{2}{*}{ Positive Religious Coping } & \multirow{2}{*}{$\boldsymbol{B}$} & $\boldsymbol{N} E$ & $\boldsymbol{T}$ & $\boldsymbol{p}$ & \multicolumn{2}{c}{ 95\% del IC } \\
\cline { 5 - 7 } & & & & & LL CI & UL CI \\
\hline Low (1.89) & 0.97 & 0.35 & 2.755 & 0.006 & 0.277 & 1.671 \\
Moderate (8.82) & 0.38 & 0.25 & 1.523 & 0.132 & -0.116 & 0.878 \\
High (15.76) & -0.21 & 0.33 & -0.638 & 0.524 & -0.867 & -0.443 \\
\hline
\end{tabular}

Note: CI: confidence interval; LL: lower limit; UL: upper limit. 


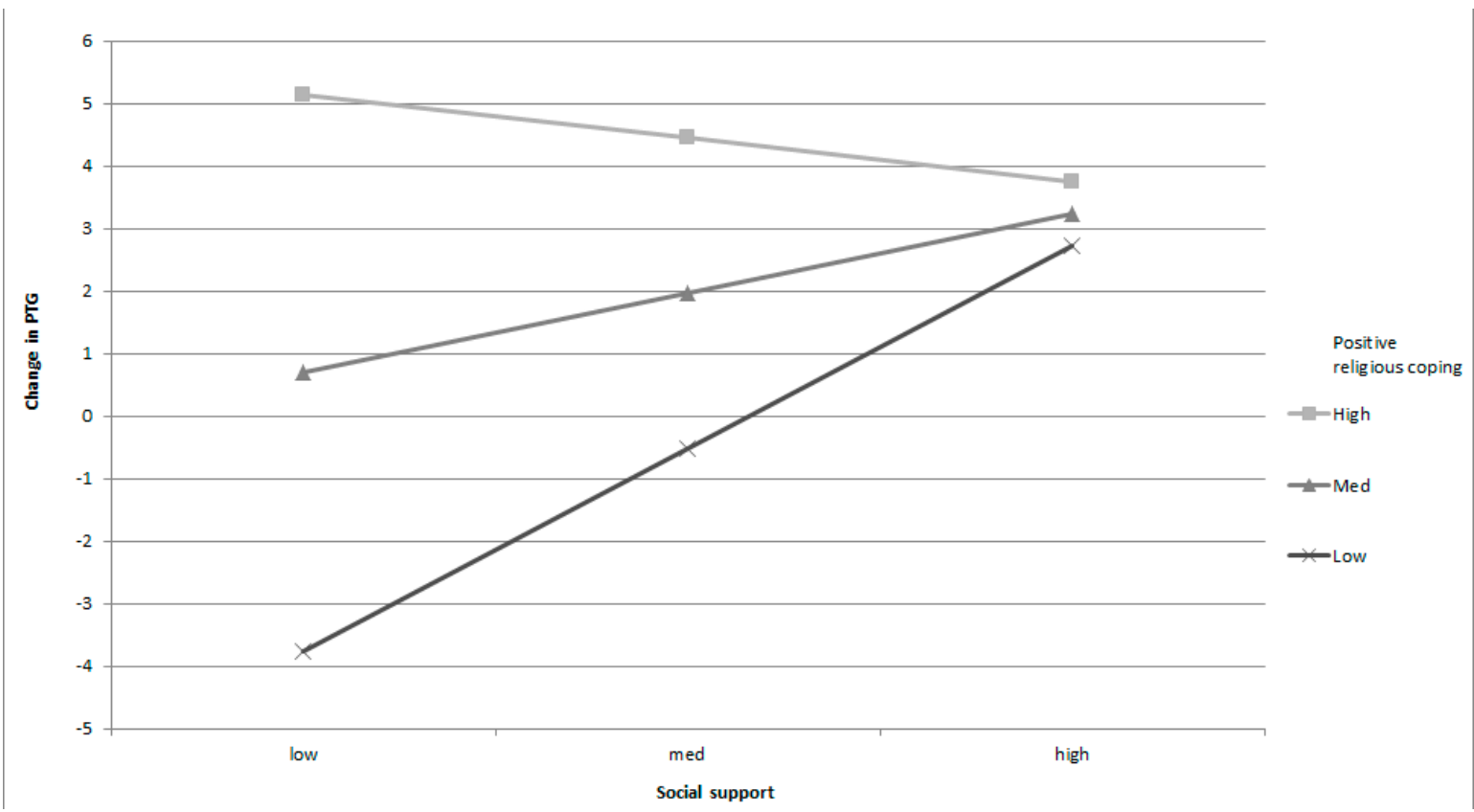

Figure 1. Change in posttraumatic growth explained by the interaction between coping by seeking social support and positive religious coping.

A second hierarchical regression evaluated the influence of RC on the change in PTS, controlling for subjective severity of event. Consequently, subjective severity was included in the first step, and positive and negative $\mathrm{RC}$ were incorporated in the second step. To evaluate a possible moderation, the interaction between subject severity and negative RC was included in a third step. The results show a significant model for the first and third steps, the third with a value $\mathrm{F}(4206)=3.384, p<0.01$; with an $R^{2}=0.06$, that is a limited amount of explained variance controlling severity, the influence of $R C$ in the increase of PTS was not significant in step 2. In step three, only interaction has significance. The results are observed in Table 5.

Table 5. Hierarchical regression on the change in posttraumatic symptoms $(n=211)$.

\begin{tabular}{|c|c|c|c|c|c|c|}
\hline & \multirow[t]{2}{*}{ Model } & \multicolumn{2}{|c|}{$\begin{array}{l}\text { Unstandardized } \\
\text { Coefficients }\end{array}$} & \multirow{2}{*}{$\begin{array}{c}\begin{array}{c}\text { Typified } \\
\text { Coefficients }\end{array} \\
\text { B }\end{array}$} & \multirow[t]{2}{*}{$T$} & \multirow[t]{2}{*}{$p$} \\
\hline & & B & SE. & & & \\
\hline \multirow[t]{3}{*}{ Step 1} & $\mathrm{R}^{2}=0.03 . \Delta \mathrm{R}^{2}=0.03 . p=0.01$ & & & & & \\
\hline & (Constant) & -1.96 & 1.20 & & -1.629 & 0.105 \\
\hline & Subjective severity T1 & 0.43 & 0.17 & 0.18 & 2.611 & 0.010 \\
\hline \multirow[t]{5}{*}{ Step 2} & $\mathrm{R}^{2}=0.04 . \Delta \mathrm{R}^{2}=0.003 . p=0.70$ & & & & & \\
\hline & (Constant) & -2.18 & 1.31 & & -1.660 & 0.098 \\
\hline & Subjective severity T1 & 0.41 & 0.17 & 0.17 & 2.381 & 0.018 \\
\hline & Positive RC T1 & 0.01 & 0.08 & 0.01 & 0.097 & 0.923 \\
\hline & Negative RC T1 & 0.12 & 0.14 & 0.06 & 0.841 & 0.401 \\
\hline \multirow[t]{6}{*}{ Step 3} & $\mathrm{R}^{2}=0.06 . \Delta \mathrm{R}^{2}=0.03 . p=0.02$ & & & & & \\
\hline & (Constant) & -0.45 & 1.48 & & -0.305 & 0.760 \\
\hline & Subjective severity T1 & 0.14 & 0.20 & 0.06 & 0.674 & 0.501 \\
\hline & Positive RC T1 & 0.03 & 0.08 & 0.03 & 0.391 & 0.696 \\
\hline & Negative RC T1 & -0.73 & 0.38 & -0.35 & -1.918 & 0.056 \\
\hline & Subjective severity $\times$ Negative $R C$ & 0.11 & 0.05 & 0.47 & 2.420 & 0.016 \\
\hline
\end{tabular}


The significant interaction of negative $R C$ and subjective severity implied the possibility of an effect of negative RC moderating the relationship between subjective severity and change in PTS. Regarding conditional effects in Table 6, the middle and high values of negative RC generated an increase of PTS for high values of subjective severity, while this does not occur when negative RC is low (see Figure 2). When negative RC is high or moderate, the severity of PTS is higher.

Table 6. Conditional effects of subjective severity in the change of posttraumatic symptoms for specific values of negative religious coping $(n=211)$.

\begin{tabular}{ccccccc}
\hline \multirow{2}{*}{ Negative Religious Coping } & \multirow{2}{*}{$\boldsymbol{B}$} & \multirow{2}{*}{$\boldsymbol{*}$} & $\boldsymbol{t}$ & $\boldsymbol{p}$ & \multicolumn{2}{c}{ 95\% del IC } \\
\cline { 5 - 7 } & & & & & LL CI & UL CI \\
\hline Low (-0.92) & 0.05 & 0.22 & 0.239 & 0.812 & -0.386 & 0.491 \\
Moderate (2.69) & 0.44 & 0.17 & 2.631 & 0.009 & 0.110 & 0.767 \\
High (6.30) & 0.82 & 0.24 & 3.428 & 0.001 & 0.350 & 1.297 \\
\hline
\end{tabular}

Note: CI: confidence interval; LL: lower limit; UL: upper limit.

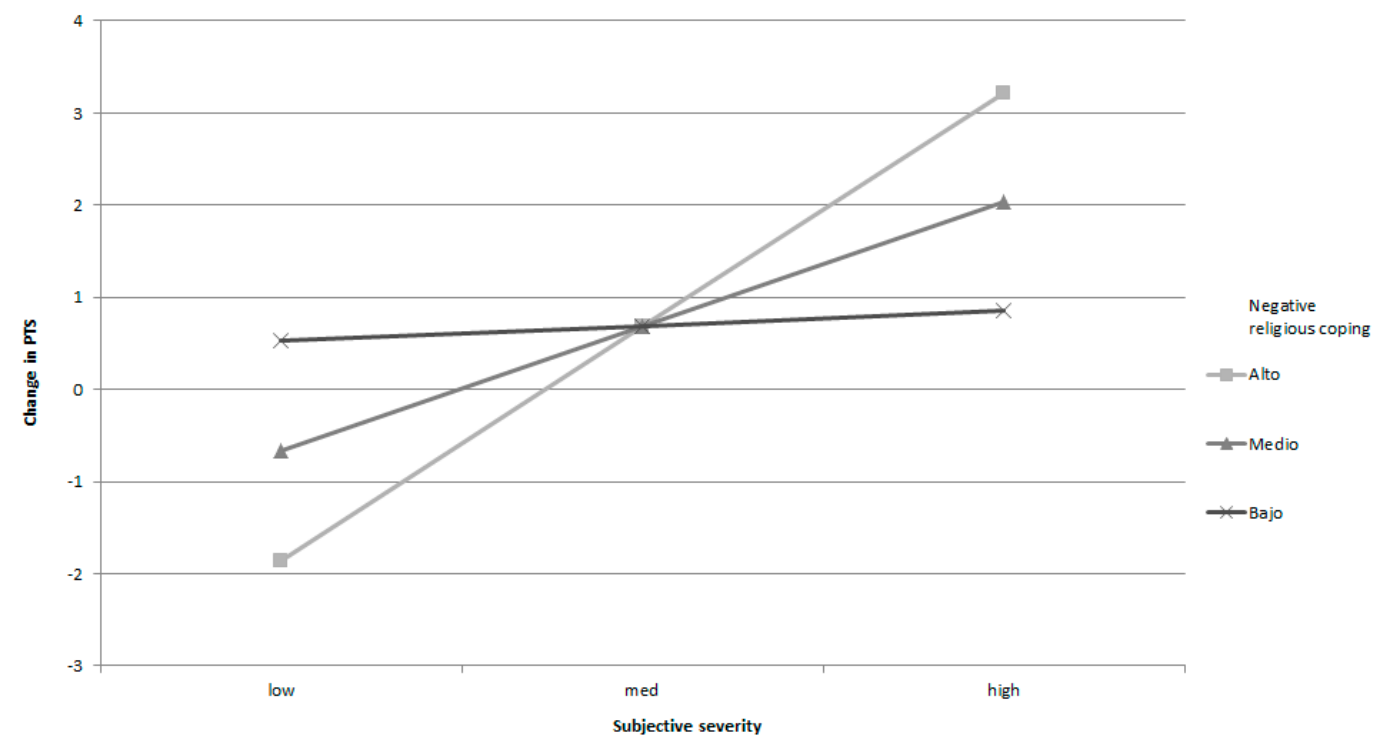

Figure 2. Change in posttraumatic symptoms explained by the interaction between subjective severity and negative religious coping.

We also performed regressions to evaluate PTG and PTS predictors at T2, controlling the influence of PTG and PTS at T1, finding similar results but with a lower effect size (data not shown).

\section{Discussion}

Results globally confirm the hypothesized associations between variables: subjective severity and negative RC affect changes to PTS more frequently, and in turn, social support and positive RC affect the changes in PTG. The positive relationship between subject severity of event and PTS had been observed in other studies [27]. While subjective severity and PTS have shown a direct relationship in studies about natural disasters [29], in this study they were not related, probably because of the more heterogeneous character of the traumatic events included.

The association of coping by social support on the increase in PTG can be explained because support networks provide conditions that favour attributing positive results to the experience [5]. On the other hand, positive religious coping can be conceived of as a form of transcendent support, or the equivalent, having the support not of a human confident but of a suprahuman one or God. One of the aspects of PTG is to value receiving and giving social support; consequently, this form of 
support would nurture this aspect. We can also suppose that receiving support helps in discovering new opportunities, changing priorities and discovering personal strengths, another three facets of PTG [3]. It is important to remark that analysis showed that high social support and positive RC, and also low negative RC, predicted PTG increase. These results are very important, because they offer longitudinal evidence about the effect of RC, which showed a specific influence controlling the coping from social support. This evidence supports the idea that religiosity helps growth beyond its function as a provider of social support networks [21]. Also, correlations and regressions showed that positive $\mathrm{RC}$ was a stronger predictor of change in PTG than social support-although the role in undermining the increase of PTG by negative RC was even lower. The direct relationship and influence of positive RC with positive change in PTG corroborates the result of other studies [8] and implies that positive $\mathrm{RC}$ would help to find a sense in life, providing a framework of meaning that helps to understand and accept the nature of stressing events and their consequences [1]. This has led to RC being considered as a healthy psychological adaptation to stress [46], providing additional benefits to other non-religious means of coping [10].

The effect of negative RC on the increase of PTS indicates that seeing god or religion as punishing or distant [31], questioning the goodness of god [24], or having doubts about the existence of a god that protects good people from bad events [47] also contributes to the increase of negative feelings associated with PTS. The predictive coefficient of RC was the lower of the three variables and its influence disappeared when severity was controlled.

Regarding the moderator role of positive RC in the relationship between social support and change in PTG, a low level of positive RC makes the relationship between social support and increase in PTG stronger. On the other hand, when positive RC is high, this relationship weakens. We must point out that a low level of positive $\mathrm{RC}$ is not the same as negative RC, but an absence of religiosity, possibly present in people of low spirituality or non-believers. In that sense, we can assume that the lack of religious coping strengthens the effect that high coping by social support gives to the increase on PTG. This result supplements the previous one and suggests that an aspect of religious coping implies obtaining social support [23]. As previously discussed, positive religious coping can be conceived of a form of transcendence support and at high level erodes the importance of secular social support.

The moderating role of negative $\mathrm{RC}$ in the relationship between subjective severity and change in the PTS also was significant. In this case, we observed that subjective severity influenced the increase of PTS only when negative RC is medium or high. These results show that religiosity does serve a moderating role between predictive variables and psychological responses after stressing events, which has been found in other studies [30,32,33], strengthening the negative impact of subjective severity when it is high. However, as Harris et al. [15] suggested, severity and Time 1 PTS could predict negative $\mathrm{RC}$ and this form of coping could act as a mediational path between baseline traumatic reactions and Time 2 PTS. In fact, they found that a proxy of severity or distress and negative RC (religious strain or conflict over faith) predicted poorer mental health after stressful life events over time. In other terms, high severity and high PTS symptoms at the base line can increase negative religious coping and by this token reinforces indirectly traumatic symptoms and decreases mental health over time. This model process is not at odds with our results, and is valid as a complementary explanation.

This study has some limitations that need to be mentioned. Firstly, the possible presence of stressful events (or other type of events that could have emerged during the period between measures) could have caused an effect over PTS and PTG that was not controlled. Secondly, the heterogeneous nature of the events to which these people were exposed is also a limitation and low degrees of freedom preclude an analysis by type of event. However, heterogeneity could also be a strength, because it represents the diversity of stressful events in the general population. On the other hand, we suppose that these results could vary in more specific samples, and we recommend continuing investigating these relationships in people exposed to more delimited stressful events. Thirdly, we do not inquire about the identification or participation of people towards a specific religious belief or whether they are agnostic or other spiritual beliefs, which prevented us from doing an analysis controlling this 
variable. Fourthly, participants reported events experienced up until four years ago, but probably a more precise baseline and follow up are needed to obtain more valid conclusions. Finally, this is a longitudinal study with only two measures, so more measures are necessary to establish the course of changes in PTS and PTG which could be non-linear and could suffer changes through time.

\section{Conclusions}

With some exceptions, the investigation about religiousness and mental health has been based on cross-sectional designs with clear limitations for causality inferences. The most important strength and contribution to the literature of this study is that it longitudinally evaluates the moderating role of religious coping on changes in posttraumatic growth and stress. Moreover, this study shows the ambivalent effect of religious coping: while positive religious coping predicts positive outcomes (the increase in PTG) negative religious coping predicts negative outcomes (the increase in PTS particularly when severity was medium high). On the other hand, this research also supports the idea that religion as a coping resource is not limited to the reception of social support, and RC shows an incremental effect above social support. Finally, RC appears as a compensatory coping reaction and at a high level neutralizes the negative effects of low social support on PTG.

The results of this longitudinal study suggest that interventions directed at incrementing positive $\mathrm{RC}$ and diminishing spiritual questioning could help reduce and prevent psychological disorders in individuals facing stressful experiences. It also suggests that seeking to increase social support is a complementary form of coping when people have neither the motivation nor socio-cognitive resources to apply positive religious coping, like individual habits and strong transcendent beliefs of prayer, or integration in a social network needed for collective religious rituals.

Acknowledgments: This work was supported by the grant CONICYT/FONDECYT 11150172 and grant UST O18872 for the first author and partially by the grant PSI2014-51923-P to the second author.

Author Contributions: Felipe E. Garcia, Darío Páez-Rovira, Alejandro Reyes-Reyes y Rodolfo Alvarez conceived the study. Felipe E. Garcia performed the statistical analysis. Felipe E. Garcia and Darío Páez-Rovira wrote the manuscript. All authors have read and approved the final manuscript.

Conflicts of Interest: The authors declare no conflict of interest.

\section{References}

1. Shaw, Annick, Stephen Joseph, and P. Alex Linley. "Religion, spirituality, and posttraumatic growth: A systematic review." Mental Health, Religion E Culture 8 (2005): 1-11.

2. Páez, Darío, Nekane Basabe, Serena Bosco, Myriam Campos, and Silvia Ubillos. "Afrontamiento y violencia colectiva (Coping and collective violence)." In Superando la Violencia Colectiva y Construyendo Cultura de Paz (Overcoming Collective Violence and Building a Culture of Peace). Madrid: Fundamentos, 2011, pp. 279-309.

3. Tedeschi, Richard G., and Lawrence G. Calhoun. "The Posttraumatic Growth Inventory: Measuring the positive legacy of trauma." Journal of Traumatic Stress 9 (1996): 455-71. [CrossRef] [PubMed]

4. Helgeson, Vicki S., Kerry A. Reynolds, and Patricia L. Tomich. “A meta-analytic review of benefit finding and growth." Journal of Consulting and Clinical Psychology 74 (2006): 797-816. [CrossRef] [PubMed]

5. Prati, Gabriele, and Luca Pietrantoni. "Optimism, social support, and coping strategies as factors contributing to posttraumatic growth: A meta-analysis." Journal of Loss and Trauma 14 (2009): 364-88. [CrossRef]

6. Urcuyo, Kenya R., Amy E. Boyers, Charles S. Carver, and Michael H. Antoni. "Finding benefit in breast cancer: Relations with personality, coping, and concurrent well-being." Psychology \& Health 20 (2005): 175-92.

7. Bosson, Julia Vigna, Mary Lou Kelley, and Glenn N. Jones. "Deliberate cognitive processing mediates the relation between positive religious coping and posttraumatic growth." Journal of Loss and Trauma 17 (2012): 439-51. [CrossRef]

8. García, Felipe E., Darío Páez-Rovira, Giordana Cartes Zurtia, Héctor Neira Martel, and Alejandro Reyes Reyes. "Religious coping, social support and subjective severity as predictors of posttraumatic growth in people affected by the earthquake in Chile on 27/2/2010." Religions 5 (2014): 1132-45. [CrossRef] 
9. Wlodarczyk, Anna, Nekane Basabe, Darío Páez, Alberto Amutio, Felipe E. García, Carlos Reyes, and Loreto Villagrán. "Positive effects of communal coping in the aftermath of a collective trauma: The case of the 2010 Chilean earthquake." European Journal of Education and Psychology 9 (2016): 9-19. [CrossRef]

10. Pargament, Kenneth, Margaret Feuille, and Donna Burdzy. "The Brief RCOPE: Current psychometric status of a short measure of religious coping." Religions 2 (2011): 51-76. [CrossRef]

11. Pargament, Kenneth I., Bruce W. Smith, Harold G. Koenig, and Lisa Perez. "Patterns of positive and negative religious coping with major life stressors." Journal for the Scientific Study of Religion, 1998, 710-24. [CrossRef]

12. Koenig, Harold G., Kenneth I. Pargament, and Julie Nielsen. "Religious coping and health status in medically ill hospitalized older adults." The Journal of Nervous and Mental Disease 186 (1998): 513-21. [CrossRef] [PubMed]

13. Fitchett, George, Bruce D. Rybarczyk, Gail A. DeMarco, and John J. Nicholas. "The role of religion in medical rehabilitation outcomes: A longitudinal study." Rehabilitation Psychology 44 (1999): 333-53. [CrossRef]

14. Pargament, Kenneth I., Harold G. Koenig, Nalini Tarakeshwar, and June Hahn. "Religious coping methods as predictors of psychological, physical and spiritual outcomes among medically ill elderly patients: A two-year longitudinal study." Journal of Health Psychology 9 (2004): 713-30. [CrossRef] [PubMed]

15. Harris, J. Irene, Christopher R. Erbes, Brian E. Engdahl, Henry Ogden, Raymond H.A. Olson, Ann Marie M. Winskowski, Kelsey Campion, and Saari Mataas. "Religious distress and coping with stressful life events: A longitudinal study." Journal of Clinical Psychology 68 (2012): 1276-86. [CrossRef] [PubMed]

16. Gerber, Monica M., Adriel Boals, and Darnell Schuettler. "The unique contributions of positive and negative religious coping to posttraumatic growth and PTSD." Psychology of Religion and Spirituality 3 (2011): 298. [CrossRef]

17. Henslee, Amber M., Scott F. Coffey, Julie A. Schumacher, Melissa Tracy, Fran H. Norris, and Sandro Galea. "Religious coping and psychological and behavioral adjustment after Hurricane Katrina." The Journal of Psychology 149 (2015): 630-42. [CrossRef] [PubMed]

18. Weber, Samuel R., and Kenneth I. Pargament. "The role of religion and spirituality in mental health." Current Opinion in Psychiatry 27 (2014): 358-63. [CrossRef] [PubMed]

19. vanOyen Witvliet, Charlotte, Karl A. Phipps, Michelle F. Dennis, and Jean C. Beckham. "Posttraumatic mental and physical health correlates of forgiveness and religious coping in military veterans." Journal of Traumatic Stress 17 (2004): 269-73. [CrossRef] [PubMed]

20. Thombre, Avinash, Allen C. Sherman, and Stephanie Simonton. "Religious coping and posttraumatic growth among family caregivers of cancer patients in India." Journal of Psychosocial Oncology 28 (2010): 173-88. [CrossRef] [PubMed]

21. Ai, Amy L., Daniel Hall, Kenneth Pargament, and Terrence N. Tice. "Posttraumatic growth in patients who survived cardiac surgery: The predictive and mediating roles of faith-based factors." Journal of Behavioral Medicine 36 (2013): 186-98. [CrossRef] [PubMed]

22. Zimet, Gregory D., Nancy W. Dahlem, Sara G. Zimet, and Gordon K. Farley. "The multidimensional scale of perceived social support." Journal of Personality Assessment 52 (1988): 30-41. [CrossRef]

23. Doane, Michael J. "The association between religiosity and subjective well-being: The unique contribution of religious service attendance and the mediating role of perceived religious social support." The Irish Journal of Psychology 34 (2013): 49-66. [CrossRef]

24. McConnell, Kelly M., Kenneth I. Pargament, Christopher G. Ellison, and Kevin J. Flannelly. "Examining the links between spiritual struggles and symptoms of psychopathology in a national sample." Journal of Clinical Psychology 62 (2006): 1469-84. [CrossRef] [PubMed]

25. Avilés Maldonado, Paola, Félix Cova Solar, Claudio Bustos, and Felipe E. García. “Afrontamiento y rumiación frente a eventos adversos y crecimiento postraumático en estudiantes universitarios (Coping and rumination against adverse events and posttraumatic growth in university students)." Liberabit 20 (2014): 281-92.

26. García, Felipe E., Carolyn Jaramillo, Ana María Martínez, Ivonne Valenzuela, and Felix Cova Solar. "Respuestas psicológicas ante un desastre natural: Estrés y crecimiento postraumático (Psychological responses to a natural disaster: Stress and post-traumatic growth)." Liberabit 20 (2014): 121-30.

27. García, Felipe E., Félix Cova, Paulina Rincón, and Carmelo Vázquez. “Trauma or growth after a natural disaster? The mediating role of rumination processes." European Journal of Psychotraumatology 6 (2015): 1-10. [CrossRef] [PubMed] 
28. García, Felipe E., Félix Cova, Paulina Rincón, Carmelo Vázquez, and Darío Páez. “Coping, rumination and posttraumatic growth in people affected by an earthquake." Psicothema 28 (2016): 59-65. [PubMed]

29. Shakespeare-Finch, Jane, and Janine Lurie-Beck. "A meta-analytic clarification of the relationship between posttraumatic growth and symptoms of posttraumatic distress disorder." Journal of Anxiety Disorders 28 (2014): 223-29. [CrossRef] [PubMed]

30. Zukerman, Gil, and Liat Korn. "Post-Traumatic Stress and World Assumptions: The Effects of Religious Coping." Journal of Religion and Health 53 (2014): 1676-90. [CrossRef] [PubMed]

31. Bradley, Rebekah, Ann C. Schwartz, and Nadine J. Kaslow. "Posttraumatic stress disorder symptoms among low-income, African American women with a history of intimate partner violence and suicidal behaviors: Self-esteem, social support, and religious coping." Journal of Traumatic Stress 18 (2005): 685-96. [CrossRef] [PubMed]

32. Thomas, Elizabeth, and Sarah Savoy. "Relationships between traumatic events, religious coping style, and posttraumatic outcomes." Traumatology: An International Journal 20 (2014): 84-90. [CrossRef]

33. Ahles, Joshua J., Amy H. Mezulis, and Melissa R. Hudson. "Religious coping as a moderator of the relationship between stress and depressive symptoms." Psychology of Religion and Spirituality 8 (2016): 228-34. [CrossRef]

34. Norris, Fran H., Jessica L. Hamblen, Lisa M. Brown, and John A. Schinka. "Validation of the Short Posttraumatic Stress Disorder Rating Interview (expanded version, Sprint-E) as a measure of postdisaster distress and treatment need." American Journal of Disaster Medicine 3 (2007): 201-12.

35. Cann, Arnie, Lawrence G. Calhoun, Richard G. Tedeschi, Kanako Taku, Tanya Vishnevsky, Kelli N. Triplett, and Suzanne C. Danhauer. "A short form of the Posttraumatic Growth Inventory." Anxiety, Stress, E Coping 23 (2010): 127-37.

36. García, Felipe E., and Anna Wlodarczyk. "Psychometric Properties of the Posttraumatic Growth Inventory-Short Form Among Chilean Adults." Journal of Loss and Trauma 21 (2016): 303-14. [CrossRef]

37. Leiva-Bianchi, Marcelo, and Ismael Gallardo Cuadra. "Validation of the short posttraumatic stress disorder rating interview (SPRINT-E) in a sample of people affected by 27-F Chilean earthquake and tsunami." Anales de Psicología (Annals of Psychology) 29 (2013): 328-34.

38. Rivera-Ledesma, Armando, and María Montero. "Espiritualidad y religiosidad en adultos mayores mexicanos (Spirituality and religiosity in Mexican elderly people)." Salud Mental 28 (2005): 51-58.

39. Carver, Charles S. "You want to measure coping but your protocol'too long: Consider the brief cope." International Journal of Behavioral Medicine 4 (1997): 92-100. [CrossRef] [PubMed]

40. Morán, Consuelo, René Landero, and Mónica Teresa González. “COPE-28: Un análisis psicométrico de la versión en español del Brief COPE (COPE-28: A psychometric analysis of the Spanish version of the Brief COPE)." Universitas Psychologica 9 (2010): 543-52.

41. Alzugaray, Carolina, Felipe E. García, Alejandro Reyes, and Rodolfo Alvarez. “Propiedades psicométricas de una versión breve de la Escala de Rumiación Relacionada a un Evento en población chilena afectada por eventos altamente estresantes (Psychometric properties of a short form of the Event Related Rumination Inventory in Chilean population affected by stressful events)." Ajayu 13 (2015): 183-98.

42. Pérez López, César, and César Pérez López. Técnicas de Muestreo Estadístico: Teoría, Práctica y Aplicaciones Informáticas (Statistical Sampling Techniques: Theory, Practice and Computer Applications). Madrid: Ra-Ma, 2000.

43. Gottman, John M., and Lowell J. Krokoff. "Marital interaction and satisfaction: A longitudinal view." Journal of Consulting and Clinical Psychology 57 (1989): 47-52. [CrossRef] [PubMed]

44. Hayes, Andrew F. Introduction to Mediation, Moderation, and Conditional Process Analysis: A Regression-Based Approach. New York: Guilford Press, 2013.

45. Bollen, Kenneth A., and J. Scott Long. Testing Structural Equation Models. Newbury Park: Sage, 1993, vol. 154.

46. Ano, Gene G., and Erin B. Vasconcelles. "Religious coping and psychological adjustment to stress: A meta-analysis." Journal of Clinical Psychology 61 (2005): 461-80. [CrossRef] [PubMed]

47. Pargament, Kenneth I. The Psychology of Religion and Coping: Theory, Research, Practice. New York: Guilford Press, 2001.

(C) 2017 by the authors. Licensee MDPI, Basel, Switzerland. This article is an open access article distributed under the terms and conditions of the Creative Commons Attribution (CC BY) license (http:/ / creativecommons.org/licenses/by/4.0/). 